\title{
L'analyse dramaturgique autochtone comme nouvel objet de la sociologie
}

\section{Indigenous Dramaturgy Analysis: A New Sociological Object El análisis de la dramaturgia autóctona como nuevo objeto de la sociología}

\section{Astrid Tirel}

Numéro 59-60, automne 2015, hiver 2016

Les nouveaux objets de la sociologie

URI : https://id.erudit.org/iderudit/1036796ar

DOI : https://doi.org/10.7202/1036796ar

Aller au sommaire du numéro

Éditeur(s)

Athéna éditions

ISSN

0831-1048 (imprimé)

1923-5771 (numérique)

Découvrir la revue

Citer cet article

Tirel, A. (2015). L'analyse dramaturgique autochtone comme nouvel objet de la sociologie. Cahiers de recherche sociologique, (59-60), 245-257.

https://doi.org/10.7202/1036796ar
Résumé de l'article

Si la sociologie peut faire son objet de tout phénomène social, elle ne peut faire abstraction des changements d'appréhension, de perception et de représentation qui interviennent entre les acteurs concernés. Or la dramaturgie autochtone nécessite un décentrement du regard pour apprécier un modèle esthétique qui, s'il s'inscrit fort bien dans les schèmes occidentaux du genre, propose une esthétique ancrée dans des savoirs culturels dont les principes et valeurs lui échappent. À ce titre, l'étude de cette dramaturgie se situe au confluent de diverses disciplines telles que les études sociologiques, anthropologiques, politiques, théâtrales, post-culturelles ou théologiques qu'elle mobilise. En bousculant la légitimité du savoir, elle exige de nouveaux outils méthodologiques susceptibles de rendre compte des réalités contemporaines de la diversité et de leur impact sur la vie en société. 


\section{L'analyse dramaturgique autochtone comme nouvel objet de la sociologie ${ }^{1}$}

AstRID TIREL

\section{Approche scientifique et spiritualité}

La sociologie, en tant que science humaine, revendique une approche scientifique qui suppose une rupture avec une construction des connaissances basée sur le religieux, sur la croyance en quelque chose qui dépasse l'humain et qui influence fortement sa condition. En quelque sorte, on pourrait dire qu'elle explique l'humain par lui-même ${ }^{2}$. Déjà Auguste Comte concevait un positivisme qui ne réduirait pas le social à des conditions extérieures à luimême, mais trouverait son sens en son propre sein ${ }^{3}$. Pour Marx, bien que de façon différente, cette rupture de l'explication de l'humain par le divin est indispensable pour amener l'Homme à penser et concevoir son propre sens

1. La dramaturgie dont il est question ici touche tant au texte qu'à la mise en scène.

2. Il ne s'agit pas ici de déconsidérer ou de hiérarchiser la place accordée par les précurseurs de la sociologie moderne à la religion, ni même de considérer leur propre athéisme ou religiosité. La religion est une part d'autant plus fondamentale de la recherche en sociologie que ses prémisses concernant I'humain ont une incidence sur la façon dont la discipline va concevoir sa propre interprétation de l'existence et sa lecture du développement des sociétés.

3. «[...] La supériorité de l'homme tient à la complexité de son organisme et non à la présence d'un principe immatériel ». Auguste Comte, cité dans Henri Gouhier, Auguste Comte, CEuvres choisies, Paris, Aubier/Montaigne, 1943, p. 35. 
et à se désaliéner de la religion qu'il considère comme «l'opium du peuple ${ }^{4}$ ». Pour ces auteurs, la religion représente une entrave au développement d'une pensée qui institue l'Homme en tant qu'élément central du monde dans lequel il évolue.

D'autres auteurs, considérés comme majeurs dans le développement de la discipline, conçoivent le religieux comme une caractéristique constitutive du sens chez les humains. Ainsi, Durkheim conçoit que la religion (ou tout au moins la foi) fait partie des schèmes de pensée des humains, en ce qu'elle leur permet de concevoir et de représenter l'intangible (comme la mort). Ce faisant, elle participe de la constitution d'une solidarité collective ${ }^{5}$. La religion est alors un des modes d'expression de l'appartenance à une société. Elle ne transcende pas le social, elle en est une composante. Weber, pour sa part, croit que la religion nourrit la rationalité propre au monde occidental ${ }^{6}$.

Ces auteurs ont formé et influencé la pensée de plusieurs générations de sociologues dans des institutions scolaires qui inscrivent l'enseignement de leurs doctrines dans l'apprentissage de base des jeunes chercheurs. Dès le Collège, les cours de méthodologie des sciences humaines offerts aux étudiants proposent une conception de la recherche scientifique qui la distingue du sens commun par la rupture avec la croyance. En ce sens, l'esprit scientifique suppose d'entretenir le doute, la certitude n'étant obtenue qu'au moyen de la preuve, qui elle-même résulte de l'expérimentation répétée. Dans le même esprit, il est enseigné que la connaissance de l'univers et sa possible transformation ne sont pas le seul apanage de Dieu, mais que les humains en sont instruits et jouent un rôle dans ce qu'est leur monde ${ }^{7}$. La démarche doit être empreinte de rationalité et le chercheur doit toujours conserver un sens critique pour se remettre en cause et remettre en cause les prémisses de sa recherche.

4. «La critique de la religion détruit les illusions de l'homme pour qu'il pense, agisse, façonne sa réalité comme un homme sans illusions parvenu à l'âge de la raison, pour qu'il gravite autour de lui-même, c'est-à-dire de son soleil réel. La religion n'est que le soleil illusoire qui gravite autour de l'homme tant que l'homme ne gravite pas autour de lui-même», Karl Marx et Friedrich Engels, Sur la religion, Textes choisis, traduits et annotés par G. Badia, P. Bange et Émile Bottigelli, Paris, Les Éditions sociales, 1968, p. 37.

5. «Une religion est un système solidaire de croyances et de Pratiques relatives à des choses sacrées, c'est-à-dire séparées, interdites, croyances et pratiques qui unissent en une même communauté morale, appelée Église, tous ceux qui y adhèrent», Émile Durkheim, Les formes élémentaires de la vie religieuse, Paris, Presses Universitaires de France, 2003, 5e édition, p. 65.

6. Max Weber, L'éthique protestante et l'esprit capitaliste, traduit par Jean-Pierre Grossein, Paris, Gallimard, 2004, 531 pages.

7. Voir Sylvain Giroux et Ginette Tremblay, Méthodologie des Sciences humaines; La recherche en action, Québec, ERPI, 2009, 324 p. [3édition], ou Valérie Blanc, Marc-André Lacelle, Christian Corno et Geneviève Perreault, IPMSHC, une approche multidisciplinaire de la recherche en sciences humaines, Montréal, Chenelière Éducation, 2010, 208 p. ou encore Maurice Angers, Initiation pra-

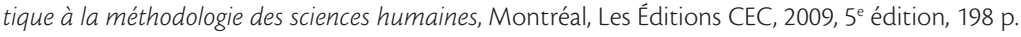


Ces principes fondamentaux de la pensée scientifique se heurtent à une lecture du monde basée sur des principes religieux. Ainsi le Christianisme, fort de plus de deux milliards de croyants, postule que Dieu a transmis son message aux humains par l'intermédiaire de Jésus, ce qui donne à la connaissance une origine divine immuable qui doit se concevoir comme une vérité. Il en est de même pour l'Islam, qui transmet à plus d'un milliard et demi de fidèles la parole de Dieu portée par Muhammad ibn Abdi Allah, parole destinée à établir des principes de vie pour les musulmans. Les spiritualités amérindiennes, quant à elles, ne répondent pas aux préceptes d'un ordre religieux institutionnalisé et pourvu d'un intermédiaire spécifique entre le monde divin et le monde humain. Mais elles répondent à des principes spirituels faisant intervenir la foi en quelque chose de plus vaste que l'humanité, qui la comprend et lui assigne un rôle de partenaire dans l'agencement du monde.

Ces conceptions spirituelles du monde se distinguent donc en apparence des principes de la recherche en sciences humaines pour qui la rationalité scientifique ne souffre pas l'argument surnaturel pour expliquer l'existence et l'organisation de vie des humains en société. Pourtant, tel qu'indiqué précédemment, la posture des précurseurs n'est pas unanime sur la question, ne serait-ce qu'en raison de leur propre éducation ou expérience respective. Cela tend à interroger, voire remettre en question, cette apparente dichotomie entre les différents points de vue, car l'écart entre l'approche scientifique et l'approche spirituelle ne semble pas outre mesure porter préjudice aux sociologues qui embrassent telle ou telle autre foi religieuse et s'accommodent fort bien des divergences de ces approches.

À travers cette incertitude quant aux statuts respectifs de la sociologie et de la religion se pose donc la question du rapport du sociologue à son objet. Cette question est majeure dans le cadre de la sociologie des religions d'obédience européenne, où le chercheur est en proie à la suspicion «parce que doublement soupçonné d'être mis au service des Églises ou à l'inverse d'être réduit au prototype de l'illusion humaine ${ }^{8} »$. Elle se pose également en sociologie de la culture où de l'art quand les analyses portant sur des pratiques à contenu spirituel vont interroger la posture du chercheur, voire interférer sur le type d'analyse qu'il va produire.

\section{Les limites de l'interdisciplinarité}

Prenons le cas du théâtre autochtone contemporain du Canada. Une description à grands traits de cet objet d'étude fera ressortir les caractéristiques

8. Pierre Lassave, «Les sociologues des religions et leur objet», Sociologie, vol. 5, n²2, 2014, p. 189. 
culturelles de ses auteurs et praticiens et la forte influence de cette empreinte identitaire sur le contenu et l'esthétique globale des pièces qui en sont issues. La spiritualité de chacune de leurs cultures est un élément constitutif de leurs traditions culturelles et leur donnent sens. La relation avec les autres habitants du Canada, qu'elle soit conflictuelle, qu'elle tende à la réconciliation ou qu'elle laisse apparaitre des espaces de partage, laisse fréquemment transparaitre une explication des différences culturelles dont une part tient à la spécificité de leurs valeurs spirituelles.

Les outils propres à la sociologie vont permettre de construire des grilles de lecture axées sur des principes critiques. Ainsi le caractère minoritaire et l'historicité de ce théâtre à prédominance culturelle enjoindront à convoquer des arguments visant à faire ressortir le rapport de domination exercé par la culture occidentale et son influence sur sa production. Il mettra également en lumière l'aliénation que ce théâtre dénonce. En ce sens, la construction de l'objet pourra, par exemple, s'inscrire dans une théorisation marxiste puis bourdieusienne dont les concepts s'appliquent à la spécificité de cet objet. Elle fera également place à la perspective postcoloniale qui, telle que formulée par Afef Benessaieh, permet de mettre en lumière des pratiques et conceptions qui échappent au regard dominant:

Il s'agit d'une perspective éminemment critique visant à corriger les biais élitistes et occidentalo-centristes des théories dominantes, en réintroduisant au centre de l'analyse des acteurs et des enjeux marginaux, invisibles ou subalternes'.

Cependant, la partie analytique d'une telle étude gagnerait à être soutenue par des disciplines ou perspectives connexes pour appréhender les références et expériences spirituelles que le théâtre autochtone invoque fréquemment à titre de spécificité culturelle. Car en effet, sans souscrire à un retour vers des valeurs spirituelles du passé, les auteurs et praticiens de théâtre autochtone revendiquent une filiation historique traditionnelle aux valeurs spirituelles qui ont guidé leurs ancêtres et se trouvent aux fondements de leurs conceptions du monde ${ }^{10}$. Figure majeure de ce théâtre au Québec, Yves Sioui exprime l'enjeu de cette revendication à travers la représentation d'une de ses pièces de théâtre:

Même pour les Indiens, voir ce spectacle (Le porteur des peines $d u$ monde) fut un choc majeur puisqu'il proposait par des images symboliques un rapatriement de la spiritualité, une reconquête de comportements archétypaux profonds liés à

9. Afef Benessaieh, «La perspective postcoloniale. Voir le monde différemment», dans Dan O'Meara et Alex McLeod (dir.), Théories des relations internationales: contestations et résistances, Montréal, Athéna éditions/Centre d'étude des politiques étrangères et sécurité (CEPES), 2010, p. 365.

10. Roberta Uno, San Pablo Burns, Lucy Mae, Jaye T. Darby (dir.), «Re-imagining the stage: tradition and transformation in Native theater», dans The color of theater: Race, culture and contemporary Performance, Continuum, 2002, p. 61 à 80. 
l'essence de la culture. De ce fait, cette production affirmait la volonté de réinsérer dans la société autochtone la fonction vitale de se représenter en tant que peuple, par le biais du théâtre ${ }^{11}$.

Ainsi la portée politique et sociale du spirituel au théâtre renseigne sur la dynamique des communautés culturelles engagées dans une réaffirmation identitaire. Mais qu'en est-il de la performativité du spirituel exprimée à travers la langue et le rituel mis en scène ? Dans le cadre d'une entrevue portant sur la réalisation de Muliats, une pièce créée par les Productions Menuentakuan, l'acteur Charles Bender évoquait la formation des acteurs prodiguée par la Compagnie Ondinnok en ces termes:

La méthode Ondinnok, c'est s'ancrer dans les objets, c'est faire un travail de masques et de personnages à travers un imaginaire typiquement autochtone, se servir de la mythologie autochtone pour aller rejoindre les personnages $[\ldots]$ On va travailler avec les pierres, on va s'ancrer dans la pierre, on va s'ancrer dans l'os qui vient de la terre, dans les ancêtres [...] on va faire des cérémonies pour se mettre dans un état de jeu. On utilise énormément la symbolique autochtone pour se créer un état de jeu qui va devenir notre état de création ${ }^{12} \ldots$

La méthode invoquée situe les référents culturels dans un animisme où l'humain, la nature et le divin vivent une étroite collaboration et donnent sens aux actions. À partir de cette approche spirituelle éloignée des conceptions occidentales qui séparent et hiérarchisent ces trois règnes, Yves Sioui construit donc une méthode de jeu basée sur des principes esthétiques qui remettent en question les approches conventionnelles occidentales. Encore faut-il être en mesure de l'appréhender, puisque cette méthode requiert une mise en condition spécifique faisant appel aux dimensions spirituelles de l'individu.

Une approche sociologique ou issue de disciplines connexes permet-elle de concevoir les modalités et l'impact de ces aspects de la performance sur les praticiens et le public? Où faut-il prendre ses distances par rapport à cette dimension de l'objet, comme le fit l'anthropologue Claude Levis Strauss à propos des mythes amérindiens?

À moi aussi sans doute, le domaine de la vie religieuse apparaît comme un prodigieux réservoir de représentations que la recherche objective est loin d'avoir épuisé; mais ce sont des représentations comme les autres, et l'esprit dans lequel j'aborde l'étude des faits religieux suppose qu'on leur refuse d'abord toute spécificité ${ }^{13}$.

11. Yves Sioui Durand cité dans Wickam Philip, «Théâtre de guérison: Entretien avec Yves Sioui Durand», Jeu, revue de théâtre, n¹13, 2004, p. 104.

12. Astrid Tirel, Propos recueillis dans le cadre d'un entretien avec le metteur en scène Xavier Huard et le comédien Charles Bender, Notes personnelles de l'auteure, 2015.

13. Claude Lévi-Strauss, Mythologiques. L'homme nu, Tétralogie IV, Plon, Paris, 1971, p. 571. 
Le fait n'empêche pas le développement d'une certaine forme de connaissance, mais appelle le questionnement suivant: lorsque le chercheur s'attache à instruire sur les sociétés et cultures étrangères à son système de constitution du savoir, ne construit-il pas des cadres d'intelligibilité limités, puisque dépourvus de la connaissance issue du milieu étudié, plus communément appelée «savoir traditionnel»?

La sociologie du théâtre s'attache volontiers aux études concernant l'acteur et le spectateur (émetteur/récepteur), le rôle que le théâtre joue dans la régulation sociale (catharsis/médiation), les conditions socio-économiques (statut/profil/subventions) des acteurs du champ ou encore la relation entre le théâtre et les institutions (propagande/transgression). Lorsqu'elle aborde la question du spirituel, cette dimension est historiquement appréhendée dans le cadre du théâtre sacré. En raison des prémisses méthodologiques de la sociologie évoquées plus tôt, sa lecture analytique entretient une dichotomie entre sacré et profane qui tend à placer derechef le chercheur dans un relativisme culturel. $\mathrm{Si}$, par le fait, il peut éviter de verser dans un certain ethnocentrisme, il peut également orienter sa quête vers un universalisme qui transcende le culturel.

Pour pallier un manque de connaissances concernant les aspects spirituels spécifiques à une culture, le sociologue ne peut se contenter des seuls bagages méthodologiques propres à sa discipline. Aussi s'ouvre-t-il aux apports de disciplines connexes plus spécialisées dans les traditions culturelles et versées dans l'étude de ces savoirs traditionnels qui lui sont moins familiers. Par le fait, il confectionne un maillage plus systématique entre les connaissances issues de sources multiples et ouvre l'interprétation sociologique à l'interdisciplinaritéél ${ }^{14}$.

La théologie aborde également le théâtre selon une perspective critique. On pensera, par exemple, à un théâtre codifié associé à la tragédie grecque dont le théâtre contemporain se serait émancipé au profit d'une sacralité endossée par le corps de l'acteur ${ }^{15}$. Dans d'autres cas, on fera référence à un théâtre d'obédience religieuse qui apparaît avec les drames liturgiques ou les Mystères. Mais encore là, l'art théâtral et la religion seront dépeints sous l'angle de leurs conflits, le premier voulant s'émanciper d'une tutelle contraignante, la seconde cherchant à s'adjoindre les modalités du premier dans une perspective utilitaire ${ }^{16}$.

\footnotetext{
14. Frédéric Darbellay, «Vers une théorie de l'interdisciplinarité? Entre unité et diversité», Nouvelles perspectives en sciences sociales, revue internationale de systémique complexe et d'études relationnelles, vol. 7, n 1, 2011, p. 65-67.

15. Anne Bouvier Cavoret (dir.), Le théâtre et le sacré, Paris, Éditions Klincksieck, 1996, 244 p.

16. Bernard Reymond, Théâtre et Christianisme, Genève, Labor et Fides, 2003, 180 p.
} 
C'est dans les formes de théâtre traditionnel non occidentales fortement empreintes de religiosité que le théâtre occidental a approché d'au plus près le spirituel. Antonin Artaud a particulièrement travaillé sur cette dimension, tentant de donner à l'acteur occidental les outils lui permettant de dépasser le psychologique pour atteindre la magie individuelle ${ }^{17}$. Artaud distingue ainsi le théâtre oriental du théâtre occidental en reconnaissant au premier moins de mots, plus de corps et une extrême codification qui se propose tel un langage symbolique à décrypter :

Une image, une allégorie, une figure qui masque ce qu'elle voudrait révéler ont plus de signification pour l'esprit que les clartés apportées par les analyses de la parole $^{18}$

À charge alors au corps en scène de traduire un langage théâtral spécifique propre à atteindre le spectateur. Artaud voit dans la proposition orientale les possibilités de transformation d'un théâtre occidental trop attaché au texte qui fige son expression et à la prépondérance d'une parole qui, trop présente, réduit ses autres formes d'expression:

Lier le théâtre aux possibilités de l'expression par les formes, et par tout ce qui est gestes, bruits, couleurs, plastique, etc., c'est le rendre à sa destinée primitive, c'est le replacer dans son aspect religieux et métaphysique, c'est le réconcilier avec l'univers ${ }^{19}$.

Et s'il ne s'attarde guère aux contextes politiques et sociaux spécifiques aux cultures desquelles il fonde sa réflexion, c'est qu'il rassemble en un seul pôle, le pôle oriental, toute la démarche des théâtres traditionnels qui gardent un lien étroit avec leurs origines religieuses :

La fascination qu'éprouve Artaud pour les cultures non occidentales ayant conservé le sens de la magie et du sacré, particulièrement au sein des rituels mais également dans leurs expressions esthétiques, le convainc que le sens véritable de

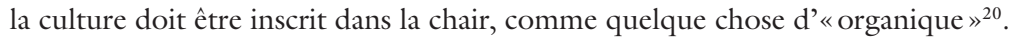

Cette conception de la culture comme élément intrinsèque du corps ouvre de nombreuses voies de recherche dans le champ théâtral. Mais elle ne permet guère de développer des outils analytiques visant à interpréter un processus spirituel en scène.

Faisant écho à l'idée d'une prépondérance des corps sur la scène, l'anthropologie théâtrale s'attache aux récurrences, au langage commun qui

17. Nägele Rainer, «L'Autre Scène: entre(nt) Brecht et Artaud», Revue de littérature comparée, $\mathrm{n}^{\circ}$ 310, 2004, p. 147-154.

18. Antonin Artaud, CEuvres, Paris, Gallimard, 2004, p. 547.

19. Ibid., p. 546.

20. Jean-François Côté, «Persona, skènè, drama: la grande transformation du théâtre et de la sociologie», Cahiers de recherche sociologique, n51, 2011, p. 105. 
constitue l'art de l'acteur, quelle que soit sa culture ${ }^{21}$. Mais là encore, cette recherche axée sur les techniques corporelles de l'individu en scène ne permet pas de rendre compte de l'immatérialité du processus spirituel et de son impact attendu en et hors scène.

L'ethnoscénologie se constitue en tant que "nouvelle discipline» rassembleuse, en 1995. Sa dynamique de recherche s'articule autour d'un travail de terrain approfondi, visant à mettre en lumière des pratiques spectaculaires méconnues du monde occidental. Son orientation à consonance démocratique et égalitariste veut donner une voie et une place légitime à l'expression singulière issue de la diversité culturelle:

Entrelaçant le descriptif et le normatif, bon nombre d'auteurs ont rendu compte de leurs perceptions à partir de modèles impropres à rendre intelligibles la complexité et la spécificité de leur objet ${ }^{22}$.

À cette fin, elle entend utiliser les méthodologies provenant d'autres disciplines, notamment «l'anthropologie du théâtre, l'ethnologie, la musicologie et la sociologie » et éviter, autant que faire se peut, les analyses ethnocentriques. Le chercheur en sciences humaines qui entend étudier le théâtre doit alors s'attacher à décrire, puis à procéder à une herméneutique de l'action. Selon Jean-Marie Pradier, la combinaison de ce processus couplée à la connaissance du performer (au sens grotowskien du terme) lui permettra d'approcher au mieux des pratiques qu'il interprète à l'aune de ses propres représentations de l'objet théâtre.

Ainsi la méthodologie propre à s'appliquer aux pratiques théâtrales dont le chercheur ignore les codes favorise l'interdisciplinarité et l'ouverture à la connaissance issue d'autres types de savoir, en l'occurrence, l'expérience du performer. Appliqué à l'étude d'un théâtre autochtone mettant en scène des performances ritualisées, ces principes méthodologiques, qui gagneraient à être développés, raisonnent singulièrement avec certains aspects caractéristiques des méthodes de recherche appliquées en milieu autochtone qui seront abordées sous peu.

Bien entendu, ces quelques disciplines attachées à la recherche théâtrale ne constituent pas, loin s'en faut, une liste exhaustive des recherches visant des pratiques spectaculaires à contenu culturel traditionnel, dont il faudrait approfondir le sens et l'ampleur pour en comprendre l'essence. Elles représentent un échantillon de disciplines qui développent des connaissances de la

21. Eugenio Barba et Nicolas Savarese, L'énergie qui danse. Dictionnaire d'anthropologie du théâtre, Montpellier, Éditions de L'Entretemps, coll. «Les voies de l'acteur», 2008.

22. Jean-Marie Pradier, "L'ethnoscénologie. Vers une scénologie générale», L'Annuaire théâtral: revue québécoise d'études théâtrales, n²9, 2001, p. 56. 
scène étayées par des principes méthodologiques sous-tendus par des paradigmes légitimés par la communauté scientifique.

Il ne s'agit donc pas de cesser d'utiliser les outils développés en recherche scientifique sous prétexte qu'ils ne répondent pas à toutes les dimensions d'un objet de recherche. Ces outils sont utiles aux penseurs, acteurs et spectateurs rompus aux codes qui leur ont été transmis dans un cadre institutionnel spécifique. En outre, Ils constituent un langage de référence intelligible, partagé et transférable.

\section{Une autre conception du savoir}

Cependant, malgré la mondialisation et sa promesse d'uniformisation, les cultures issues d'autres traditions de pensée introduisent d'autres voix, d'autres expériences, d'autres expertises et d'autres perspectives dans le savoir occidental dominant. La justification, voire la légitimité de l'usage exclusif du savoir scientifique en recherche, est interrogée en raison de la transformation du profil des chercheurs et de l'introduction de nouveaux objets de recherche. Au Canada, une intensification de la mobilité intellectuelle et l'accessibilité au milieu universitaire favorisent le développement d'une diversité culturelle qui prête à la rencontre de points de vue différents. Ces autres manières d'appréhender le savoir bousculent les prémisses sur lesquelles se base la recherche scientifique dominante en l'obligeant à reconsidérer son propre processus de constitution du savoir. Les pressions exercées par la communauté internationale pour la reconnaissance de la diversité culturelle et la mise en place de programmes visant à soutenir concrètement l'expression de la diversité participent de cette transformation ${ }^{23}$.

Les sciences de la santé, les sciences politiques, les sciences de l'éducation et les sciences de l'environnement connaissent un intérêt marqué pour les savoirs autochtones ${ }^{24}$. Ces savoirs, appliqués aux recherches concernant les peuples autochtones eux-mêmes, s'avèrent plus à même de servir les intérêts des membres des communautés, et sont potentiellement porteurs de

23. Déclaration universelle de I'UNESCO sur la diversité culturelle (2001), Priorité sur la recherche autochtone par le Conseil de recherche en Sciences humaines (2002), Convention pour la sauvegarde du patrimoine culturel immatériel, UNESCO (2003); Déclaration des Nations Unies des Droits des Peuples Autochtones (2007).

24. Manuela Carneiro da Cunha, Savoirs autochtones: quelle nature, quels apports? Leçon inaugurale prononcée le jeudi 22 mars 2012 dans Savoirs autochtones: quelle nature, quels apports? Leçon inaugurale prononcée le jeudi 22 mars 2012 [http://books.openedition.org/cdf/1286?lang=fr], Paris, Collège de France, 31 p.; Djakalidja Coulibaly, «Développement durable et savoirs autochtones: une nouvelle perspective pour les sciences sociales», European Scientific Journal, vol. 8, n 26, 2012, p. 66-76; Arun Agrawal, «Classification des savoirs autochtones: la dimension politique», Revue internationale des sciences sociales, vol. 3, $n^{\circ} 173,2002$, p. 325-336. 
connaissance à l'échelle internationale. Mais l'accès à ces savoirs et la manière d'en faire usage se heurtent à leur moindre adéquation avec les méthodes scientifiques. Aussi, les limites de l'approche scientifique l'enjoignent à se familiariser avec les méthodologies issues d'autres traditions de pensée, en l'occurrence, les méthodologies issues du terrain de recherche.

Carolyn Kenny et ses collaboratrices ont produit, en 2004, un document commandé par Condition féminine Canada afin de constituer une référence pour les chercheurs autochtones et non autochtones ${ }^{25}$. Le rapport propose une approche holistique qui suppose de combiner tradition et modernité dans la recherche. S'il s'attache à apporter un éclairage sur la place et le rôle des femmes dans les cultures autochtones, il offre plus largement un cadre de référence permettant d'aborder les processus et enjeux menant au développement du savoir dans les communautés. Selon les chercheuses, les savoirs traditionnels s'élaborent autour de trois pôles qui constituent l'approche holistique à respecter, à savoir, l'histoire (passée, présente et future), le rapport au monde (l'interdépendance) et les différentes dimensions qui constituent l'humain (le spirituel, le physique, l'émotif et le mental). L'approche holistique constitue un aspect majeur des caractéristiques associées aux savoirs autochtones qui les distinguent des savoirs occidentaux ${ }^{26}$.

La confrontation entre savoirs scientifiques et savoirs traditionnels force à reconsidérer le discours sur la nature du savoir, tandis que le travail de coopération en recherche permet de connaitre l'approche autochtone et son effectivité dans la production des connaissances. Cette approche se construit au regard des contextes historique, expérientiel, situationnel et projectif impliqués dans les savoirs issus des communautés. Aussi, la connaissance de l'histoire coloniale, le rapport des membres des communautés avec les tenants de la recherche scientifique occidentale, la connaissance du milieu, le niveau de vie et les représentations de l'avenir constituent le cadre contextuel

25. Carolyn Kenny et al., Cadre holistique pour la recherche en matière de politiquesautochtones, 2004 [http://publications.gc.ca/site/fra/263859/publication.html].

26. Notons que si les savoirs traditionnels autochtones évoluent dans un cadre holistique, leurs modes d'acquisition sont divers. Ils peuvent, par exemple, être le fruit d'une recherche personnelle et des expériences menées par un chaman qui les délivrera à la communauté ou encore être la résultante d'une entreprise collective et se perpétuer à travers les mythes cosmogoniques transmis de génération en génération. Voir l'article de Manuela Carneiro da Cunha, op. cit. Dans ce même article, l'auteure évoque également la pluralité du terme «savoirs traditionnels»: «J'insiste sur le pluriel car on n'est pas en présence d'un seul mode d'accès à la connaissance et d'un seul régime, mais bien d'une pléthore qu'il faut encore apprendre à connaître» (par. 40). L'expression fait d'autant plus débat qu'elle engendre une réflexion visant à circonscrire quels savoirs sont «traditionnels » ou non, en raison de l'implication politique, économique et juridique que cela entraîne. Voir Alain Pierre-Collot, «La protection des savoirs traditionnels, du droit international de la propriété intellectuelle au système de protection sui generis », Droit et cultures, 2009 [http://droitcultures.revues. org/502]. 
de la recherche partenariale avec les peuples autochtones. La connaissance du présent ancrée dans l'histoire, permet de percevoir la transformation des rapports et des valeurs entre les membres des communautés, à partir de l'étude de l'organisation des sociétés passées et des effets du changement opéré sur l'équilibre des sociétés, à partir de la colonisation.

Cette prise en compte des savoirs traditionnels autochtones et de leur contexte d'expression contemporain a l'avantage de ne pas figer les savoirs traditionnels dans un passé mythique. Elle permet également de considérer le fait que différents «régimes de savoir» peuvent se côtoyer et contribuer à l'avancement des connaissances ${ }^{27}$.

Pour un sociologue, la recherche ne se mène plus seule dans un rapport linéaire de type chercheur/répondant, mais fait l'objet d'un travail collectif. Son statut et son rôle ne sont plus déterminés par les seules instances académiques dont il dépend. Il devient un collaborateur à la recherche tandis que l'apport et l'implication des personnes concernées par la recherche en font des partenaires. Ces transformations du rôle de chacun inscrit la relation entre Autochtones et non-Autochtones dans une perspective plus égalitaire que le rapport d'ascendance de la communauté scientifique aux savoirs traditionnels ne le faisait. Ce faisant, cette démarche collaborative, parfois qualifiée de «révolution épistémologique s'inscrit dans le vaste mouvement de démocratie participative que la reconfiguration politique internationale semble exiger ${ }^{28}$.

Mais elle ne va peut-être pas aussi loin que ce qui est attendu par les instances politiques autochtones dont le projet de décolonisation du savoir parait plus ambitieux. Comme l'indique le document produit par le Conseil de recherches en sciences humaines du Canada (le CRSH), ce changement de paradigme en recherche émane pour beaucoup du Saskatchewan Indian Federated College (SIFC) ${ }^{29}$ dont le mémoire produit en 2002 prend très nettement position pour une réappropriation du savoir concernant les populations autochtones par elles-mêmes. Le propos fait écho à la proposition de principe émise, en 1972, par la Fraternité indienne du Canada à l'attention du ministre des Affaires indiennes et du Nord canadien intitulée «La maittrise indienne de l'éducation indienne». Aussi, le partenariat s'avère une réponse politique salutaire pour répondre aux idéaux démocratiques revendiqués par les sociétés occidentales et par les exigences d'application de ces mêmes idéaux démocratiques par les peuples autochtones.

27. Manuela Carneiro da Cunha, op. cit.

28. Marie Roué, «Histoire et épistémologie des savoirs locaux et autochtones», Revue d'ethnoécologie, 2012 [http://ethnoecologie.revues.org/813].

29. Aujourd'hui, Université des Premières Nations du Canada. 
Sans entrer plus avant dans les considérations politiques que cela implique, notons que ce mouvement de rééquilibrage des relations entre les chercheurs occidentaux et les peuples autochtones invitent la sociologie à une refonte de ses principes éthiques ${ }^{30}$. Car en plus de la reconnaissance des savoirs traditionnels autochtones, il lui faut prendre en compte la pluralité des appartenances culturelles de ses chercheurs dont les orientations et les objets de recherche vont lentement transformer la discipline, comme le processus de reconnaissance des savoirs autochtones va immanquablement les transformer ${ }^{31}$.

Aussi, et pour en revenir à notre objet, l'analyse d'une pièce de théâtre construite à l'aide d'éléments traditionnels a avantage à tenir compte des facteurs spirituels et contextuels spécifiques à la culture afférente, dans la mesure où ces éléments informent au-delà des procédés conventionnels convoqués par un public de théâtre non autochtone. Or, une meilleure compréhension du théâtre autochtone passe par une réception informée de son esthétique.

Ainsi, la portée d'un mythe autochtone engagé dans la dramaturgie d'une pièce de théâtre, telle que Le Maître de la Rosée $e^{32}$, peut revêtir une signification supplémentaire à la lecture psychanalytique qui nous renseigne sur les tourments des personnages ou la transposition politique qui éclaire les enjeux nationaux et internationaux à l'œuvre. Elle invite à reconsidérer les catégories associées à des genres littéraires spécifiques tels que la littérature enfantine, la littérature jeunesse ou la littérature fantastique - genres que l'on pourrait apposer à cette pièce en raison de son inscription dans un univers mythique où les animaux comme les humains sont doués de parole - pour envisager l'esthétique globale de l'oeuvre ${ }^{33}$. La pièce de Floyd Favel s'inscrit dans les principes de perpétuation des savoirs traditionnels autochtones. La pièce est construite à partir d'un conte dont le récit lui a été fait par une aînée, sa mère, auquel il a ajouté une interprétation personnelle. Son contenu vise à faire connaitre les relations entre Autochtones et non-Autochtones depuis la colonisation. De

30. Il s'agit de réfléchir au principe d'objectivité du chercheur en fonction de son implication et des appartenances ainsi qu'aux limites de cette objectivité. Voir Pierre Bourdieu, «Sociologie de la croyance et croyance des sociologues », Archives de sciences sociales des religions, vol. 63, n 1, 1987, p. $155-161$.

31. Agrawal Arun, «Classification des savoirs autochtones: la dimension politique», Revue internationale des sciences sociales, vol. 3, no 173, 2002, p. 325-336.

32. Il s'agit de l'adaptation de la pièce de Floyd Favel, dont le titre original en anglais est Governor of the Dew (1996). Au Québec, la pièce a été traduite en français par Jean-Frédéric Messier et mise en scène par Catherine Joncas, pour Ondinnok. Le propos concernant cette pièce touche davantage la création même de l'œuvre et le contexte de cette création que sa version écrite ou mise en scène en anglais ou en français.

33. L'esthétique s'entend ici selon la définition qu'en donne Catherine Naugrette: «Ce sont désormais non seulement les conditions d'apparition de l'œuvre, sa nature ou ses significations, mais aussi l'expérience du créateur comme celle du récepteur que l'esthétique doit prendre en charge», Catherine Naugrette, L'esthétique théâtrale, Paris, Armand Collin, 2010, p. 19. 
la sorte, l'histoire de la rencontre et de la relation entre les Européens venus conquérir l'Ouest canadien et les Cris se perpétue d'une génération à l'autre et constitue un savoir actuel.

Une pièce comme Conte d'un indien urbain ${ }^{34}$, enrichie d'un contenu spirituel dans la mise en scène proposée au Québec, apporte une touche de rituel performative en investissant le corps du comédien d'un état d'être destiné à provoquer une communication avec d'autres êtres, à travers les pierres dont il se saisit et qu'il déplace sur la scène. Pour le milieu théâtral autochtone, cette perspective est gage de légitimité. Elle permettrait de développer et de transmettre des schèmes de pensées dont la codification serait plus familière au public, ce qui lui ferait gagner en visibilité et en intérêt.

Par ailleurs, une telle initiative endossée par la sociologie ouvrirait la perspective de participer à la transformation du socius en modifiant les mentalités de son propre milieu. Car, se sensibiliser et s'ouvrir aux perspectives autochtones permettraient de contribuer davantage à une réelle rencontre entre Autochtones et non-Autochtones qui partagent un milieu commun. Il s'agit, en somme, d'entretenir cette ouverture d'esprit qui caractérise la recherche scientifique tout en étant sensible aux transformations de la société qui fait de tout sujet ou groupe autochtone un partenaire à part entière de notre accès au savoir.

De plus, l'exercice vise à s'assurer, par la remise en question des méthodes sociologiques conventionnelles, de leur viabilité ou de leur obsolescence dans la compréhension d'objets qu'elle connaît moins et possiblement de réviser les méthodes sociologiques pour maintenir leur pertinence. Enfin, affiner la compréhension du théâtre autochtone, en tant que nouvel objet de la sociologie est un acte potentiellement porteur de clés de lecture ouvrant sur de nouveaux horizons, tant sur le plan de l'art théâtral que sur celui de la société qui le produit.

34. Pièce de Darrel Dennis, mise en scène par Catherine Joncas pour Ondinnok. 